\title{
ON BIEBERBACH-EILENBERG FUNCTIONS. II
}

\author{
BY
}

JAMES A. JENKINS

1. Let us denote by $C$ the class of functions $f(z)$ regular for $|z|<1$ which have power series expansion about $z=0$ beginning

$$
f(z)=a_{1} z+a_{2} z^{2}+\cdots
$$

and which satisfy the condition

$$
f\left(z_{1}\right) f\left(z_{2}\right) \neq 1, \quad\left|z_{1}\right|<1, \quad\left|z_{2}\right|<1 .
$$

Let $C(\lambda)$ denote the subclass of $C$ consisting of functions $f(z)$ for which $\left|a_{1}\right|=\lambda, 0<\lambda \leqq 1$. If $f \in C(\lambda)$ it is subordinate to a function $g(z) \in C$ univalent in $|z|<1$ [9] for which then $\left|g^{\prime}(0)\right| \geqq \lambda$. Thus the image of $|z|<1$ by $w=g(z)$ covers the circle $|w|<\lambda / 4$ and hence by condition (1), for $|z|<1,|g(z)|$ $<4 / \lambda$. Consequently, for $|z|<1,|f(z)|<4 / \lambda$ so that the functions in $C(\lambda)$ are uniformly bounded. Rogosinski [9] raised the question of the best possible bound $P(\lambda)$ for the functions in $C(\lambda)$. He gave certain upper estimates for $P(\lambda)$ but not the precise value. The object of the present paper is to give the complete solution of this problem by a method used earlier to solve certain other questions for the same class of functions [5].

2. We begin by solving first the related problem of the closest boundary point to the origin of the image of $|z|<1$ by a univalent function in $C(\lambda)$. In the $\zeta$-plane $(\zeta=\xi+i \eta)$ we regard the domain $\Delta(t)$ defined by

$$
\begin{aligned}
0<\eta<\pi, & \xi<0, \\
0<\eta<\pi-t, & \xi=0, \\
-t<\eta<\pi-t, & \xi>0,
\end{aligned}
$$

where $0 \leqq t<\pi$. Let us denote the following boundary points of $\Delta(t)$ in the manner indicated: $0, B ;-i t, C ; i(\pi-t), E ; \pi i, F$. Further let the boundary point of $\Delta(t)$ at infinity between $F$ and $B$ in the natural cyclic order be denoted by $A$, the corresponding point between $C$ and $E$ by $D$. We map $\Delta(t)$ conformally onto the left-hand half-plane $\Im w<0$ in such a way that $A$ goes into $w=0, D$ into $w=\infty$. Let us denote by $P$ the point $\zeta=(\pi-t) i / 2$. Rotation of $\Delta(t)$ through $180^{\circ}$ about $P$ corresponds in the $w$-plane to a linear transformation of $\Im w<0$ on to itself interchanging 0 and $\infty$. This transformation has the form $w^{*}=a / w, a$ real and positive. The fixed point $-a^{1 / 2}$ (positive root) of the latter transformation is the image of $P$. We adjust the original mapping from the $\zeta$-plane to the $w$-plane so that this becomes the point -1 and the linear transformation becomes $w^{*}=1 / w$.

Received by the editors May 10, 1954. 
Let the images of $B$ and $C$ be $i / l$ and $i / m, l \geqq m>0$. Then the images of $E$ and $F$ are $-i l,-i m$. If we extend $\zeta$ as a (non-single-valued) function of $w$ to the whole $w$-plane by reflection in various segments of the imaginary axis we see at once that $d \zeta^{2}$ is a quadratic differential on the $w$-sphere with double poles at $0, \infty$, simple poles at $i / m,-i m$ and simple zeros at $i / l,-i l$ (except in the case $t=0$ when the simple poles and zeros coincide in pairs and cancel). Indeed we can write

$$
d \zeta^{2}=K \frac{(w+i l)(w-i / l)}{w^{2}(w+i m)(w-i / m)} d w^{2}
$$

with $K$ a suitable positive constant. In case $t=0$ this assumes a simple limiting form. The curves on which $d \zeta^{2}>0$ will be called trajectories, those on which $d \zeta^{2}<0$ will be called orthogonal trajectories.

Let us regard now the mapping from the domain $|z|<1, \Re z<0$ in the $z$-plane onto the domain $0<\eta<\pi, \xi<0$ in the $\zeta$-plane by the function $\zeta=\log (z / i)$ (with a suitable determination). Combining this with the above mapping from the $\zeta$-plane into the $w$-plane and extending it by reflection across the segments joining $i$ and $-i$ in the $z$-plane and $i / l$ and $-i m$ in the $w$-plane we obtain a function regular and univalent for $|z|<1$. We denote this function by $f(z ; t)$ and the image of $|z|<1$ under it by $D(t)$. The latter domain is bounded by orthogonal trajectories joining $i / l$ and $-i l$ and a rectilinear slit along an orthogonal trajectory from $-i m$ to $-i l$. The latter slit degenerates to a point when $t=0$. Further $f(z ; t) \in C$ since the transformation $w^{*}=1 / w$ carries $D(t)$ into its exterior; also $f^{\prime}(0 ; t)>0$ and $f(z ; 0) \equiv z$.

The quantities $m$ and $l$ depend on the parameter $t$ and when appropriate will be denoted by $m(t)$ and $l(t)$.

3. Theorem 1. Let $g(w)$ be regular and univalent in $D(t)$ with $g(0)=0$, $\left|g^{\prime}(0)\right|=1$ and such that $g\left(w_{1}\right) g\left(w_{2}\right) \neq 1, w_{1}, w_{2} \in D(t)$. Let $\mu$ denote the modulus of the boundary point of the image of $D(t)$ under $w^{\prime}=g(w)$ closest to $w^{\prime}=0$. Then $\mu \geqq m(t)$ and equality can be obtained only for $g(w)= \pm \phi(w)$, provided $t>0$, where $\phi(0)=0$ and $\phi$ is a conformal mapping of $D(t)$ upon itself. When $t=0$, equality is attained only for $g(w)=e^{i \theta} \phi(w), \theta$ real, $\phi$ as before.

The proof of this result depends on the consideration of the following module problem. Let $L$ be a Jordan curve in the $w$-plane enclosing $w=0$ and having reflectional symmetry in the imaginary $w$-axis. Let $L^{*}$ be the image of $L$ under the transformation $w^{*}=1 / w$. We suppose that $L^{*}$ is exterior to $L$ and denote by $\mathfrak{D}$ the doubly-connected domain bounded by $L$ and $L^{*}$. Let $G$ be a point on the positive imaginary axis in $\mathfrak{D}$ and $G^{*}$ its image under the transformation $w^{*}=1 / w$. Let $C_{1}$ denote the class of rectifiable Jordan curves lying in $\mathfrak{D}$ and separating $L$ from $G, G^{*}$, and $L^{*}$. Let $C_{2}$ denote the class of rectifiable Jordan curves lying in $\mathfrak{D}$ and separating $L^{*}$ from $L, G$ and $G^{*}$. 
Let $\rho$ be a real-valued non-negative function of integrable square over $\mathfrak{D}$ and such that, for $c \in C_{i}(i=1,2), \int_{c} \rho|d w|$ exists and that $\int_{c} \rho|d w| \geqq 1$. Then let the greatest lower bound of $\iint_{D \rho^{2}} d u d v(w=u+i v)$ for all such functions $\rho$ be denoted by $M(L, G)$. This actually is a minimum attained for a particular function $\rho$. This can be proved by reducing the problem to a hexagon problem by a method similar to that of [2] or by a general construction method, but this result is not needed here.

Let us return now to the quadratic differential $d \zeta^{2}$ which we denote by $Q(w) d w^{2}$. We have seen that $D(t)$ is bounded by the union of certain orthogonal trajectories. The orthogonal trajectories interior to $D(t)$ are Jordan curves with reflectional symmetry in the imaginary $w$-axis. The orthogonal trajectory of this set which meets the positive imaginary axis at the point ir $(r>0)$ will be denoted by $H(r)$. As $r$ tends to zero $H(r)$ tends to circular form $[10 ; 6]$. Let $H(r)$ for $r$ sufficiently small play the role of $L$ in the preceding module problem and let the point $w=i / m$ play the role of $G$. Let the doubly-connected domain bounded by $H(r)$ and the boundary of $D(t)$ be denoted by $E(r)$ and its image under the transformation $w^{*}=1 / w$ be denoted by $E^{*}(r)$. These two domains have equal module (for the class of curves separating the boundaries) which we denote by $M(r)$. We then verify readily that for a suitable constant $\nu$ the metric $\nu|Q(w)|{ }^{1 / 2}|d w|$ provides the extremal metric in the module problem defining $M(L, G)$ and this independently of the value of $r$. Further, in this case, $M(L, G)=2 M(r)$.

With again the choice $L=H(r)$ but for a point $H$ with affix $i h, h>1 / m$, we have $M(L, G) \geqq M(L, H)+d$ where $d(>0)$ is independent of $r$ but depends on $h$. This can be seen in various ways, perhaps most easily by observing that it is possible to modify the function $|Q(w)|^{1 / 2}$ by setting it equal to zero in a sufficiently small neighborhood of $G$ to obtain a function admissible in the competition for the greatest lower bound $M(L, H)$, independent of the choice of $r$.

Let now $E^{\prime}(r)$ be the image of $E(r)$ under the function $g$ of Theorem 1 . We shall suppose it to lie again in the $w$-plane. Let $K_{1}$ be the bounded continuum complementary to $E^{\prime}(r)$ bounded by the image $L^{\prime}$ of $L$. Let $K_{2}$ be the other continuum complementary to $E^{\prime}(r)$. We obtain from $E^{\prime}(r)$ a circularly symmetrized domain $\tilde{E}(r)$ in the following manner. Let the intersections of $|w|=R$ with $K_{1}$ and $K_{2}$ have respectively angular Lebesgue measure $l_{1}(R)$ and $l_{2}(R)$. Let $\widetilde{K}_{1}$ be the set defined by $\pi / 2-l_{1}(R) / 2 \leqq \Phi \leqq \pi / 2+l_{1}(R) / 2$ for those values of $R$ for which $K_{1}$ meets $|w|=R$ where $R, \Phi$ are polar coordinates in the $w$-plane. Let $\tilde{K}_{2}$ be the set defined by $-\pi / 2-l_{2}(R) / 2 \leqq \Phi \leqq-\pi / 2$ $+l_{2}(R) / 2$ for those values of $R$ for which $K_{2}$ meets $|w|=R$. The complement of $\widetilde{K}_{1} \cup \widetilde{K}_{2}$ is a doubly-connected domain which we denote by $\tilde{E}(r)$.

Let $E^{\prime}(r), \tilde{E}(r)$ have modules $M^{\prime}(r), \tilde{M}(r)$. Clearly $M^{\prime}(r)=M(r)$ while by a standard symmetrization argument $M^{\prime}(r) \leqq \tilde{M}(r)$ [8]. Under the assumption of Theorem 1 the point of $K_{2}$ closest to $w=0$ has modulus $\mu$, thus the 
point $-i \mu$ is a point of $\tilde{K}_{2}$ and a boundary point of $\tilde{E}(r)$. We verify at once that $\tilde{E}(r)$ does not overlap with its image $\tilde{E}^{*}(r)$ under the transformation $w^{*}=1 / w$ (as a consequence of the condition $g\left(w_{1}\right) g\left(w_{2}\right) \neq 1, w_{1}, w_{2} \in D(t)$ ). Let us now denote the point $i / \mu$ by $H$ and let $E(r)$ be the intersection of $E(r)$ with the exterior of $L$. Let this domain, which is doubly-connected at least for $r$ small enough, have module $\mathfrak{M}(r)$ (for the class of curves separating its boundaries as usual). Since $H(r)$ tends to circular form as $r$ tends to zero we have that $\tilde{M}(r)-\mathfrak{M}(r)$ approaches zero as $r$ tends to zero. We see also that $\&(r)$ separates $L$ from $H, H^{*}$ and $L^{*}$, and $\mathbb{F}^{*}(r)$, its image by the transformation $w^{*}=1 / w$, separates $L^{*}$ from $L, H$ and $H^{*}$.

Suppose now that $\mu<m(t)$. Then, by an earlier remark, $M(L, G)$ $\geqq M(L, H)+d$ where $d(>0)$ is independent of $r$. On the other hand, by a standard argument $[4], M(L, H) \geqq 2 \mathfrak{M}(r)$. Thus, combining this with the statements $M(L, G)=2 M(r), \tilde{M}(r)-\mathfrak{M}(r)=o(1)$, we have

$$
2 M(r) \geqq 2 \tilde{M}(r)+d+o(1),
$$

a contradiction to the result $\tilde{M}(r) \geqq M(r)$. Thus $\mu \leqq m(t)$.

Suppose next that $\mu=m(t)$. If $\widetilde{K}_{2}$ did not coincide with the complement of $D(t)$ it would follow by a standard form of argument [3] that we would have $M(r) \geqq \tilde{M}(r)+\delta+o(1)$ for $\delta(>0)$ independent of $r$ for $r$ sufficiently small. This would be in contradiction to the inequality $\tilde{M}(r) \geqq M(r)$. Further, unless $\widetilde{K}_{2}$ is obtained from $K_{2}$ by a rigid rotation about $w=0$, we shall have $\tilde{M}(r) \geqq M(r)+p$ where $p$ is a positive constant independent of $r$, for $r$ sufficiently small [7, Theorem 3$]$. In this case we would have $M(L, G) \geqq 2 \mathfrak{M}(r)$ and we would get

$$
2 M(r) \geqq 2 \tilde{M}(r)+o(1)
$$

contrary to the inequality $\tilde{M}(r) \geqq M(r)+p$.

Thus equality is possible at most when $g(w)$ has the form $e^{i \theta} \phi(w), \theta$ real, with $\phi(w)$ a conformal mapping of $D(t)$ onto itself and $\phi(0)=0$. The function $e^{i \theta} \phi(w)$ can satisfy the conditions of Theorem 1 only if neither 1 or -1 is interior to the image of $D(t)$ by this function. Let us assume first that $t>0$. Then the only boundary points of $D(t)$ on $|w|=1$ are the points $w= \pm 1$. This can be verified by observing that $|w|=1$ has the direction of an orthogonal trajectory only at the points $w= \pm i$ and that this situation will occur between any two boundary points on $|w|=1$. That $d \zeta^{2}<0$ on $|w|=1$ only at $w= \pm i$ is the consequence of a simple direct numerical calculation. Thus the open $\operatorname{arc}|w|=1, \Im w>0$ is interior to $D(t)$, the open $\operatorname{arc}|w|=1, \Im w<0$ is exterior to $D(t)$ and the only values of $e^{i \theta}$ for which $e^{i \theta} \phi(w)$ satisfies the conditions on $g(w)$ in Theorem 1 are \pm 1 . When $t=0$ all values $e^{i \theta}, \theta$ real, are clearly admissible. This completes the proof of Theorem 1 .

4. The uniqueness part of Theorem 1 implies that no two values $f^{\prime}(0 ; t)$ are equal. By an argument involving the theory of normal families we see that 
$f^{\prime}(0 ; t)$ tends to zero as $t$ approaches $\pi$. Also for $t=0, f^{\prime}(0 ; 0)=1$. Thus as $t$ takes the values $0 \leqq t<\pi, f^{\prime}(0 ; t)$ takes the values $1 \geqq f^{\prime}(0 ; t)>0$. In particular for $1 \geqq \lambda>0$ there is a unique such function with $f^{\prime}(0 ; t)=\lambda$. We denote this function by $F(z ; \lambda)$ and the modulus of the closest boundary point to the origin of the image of $|z|<1$ under this function by $\mu(\lambda)$. Clearly $F(z ; \lambda)$ $\in C(\lambda)$. We then have the following result.

THEOREM 2. If $f(z)$ is a univalent function in $C(\lambda)$, then for the modulus $\mu$ of the boundary point of the image of $|z|<1$ under $f$ closest to the origin we have $\mu \geqq \mu(\lambda)$ and equality can be attained only for $f(z)= \pm F\left(e^{i \psi} z ; \lambda\right), \psi$ real, when $\lambda<1$ and for $f(z)=e^{i \theta} z, \theta$ real, when $\lambda=1$.

This is an immediate consequence of Theorem 1.

5. We now turn to the determination of $P(\lambda)$, the best possible uniform bound for functions in $C(\lambda)$. We observe first that we obtain the same result if we restrict ourselves to univalent functions in $C(\lambda)$. Indeed $f(z) \in C(\lambda)$ is subordinate to a univalent function $g(z) \in C$ [9]. The function $g(z)$ will not in general be in $C(\lambda)$ but $\left|g^{\prime}(0)\right| \geqq \lambda$. Thus, producing a slit from the boundary of the image under $g$ of $|z|<1$ toward the origin and letting $h(z)$ be a function mapping $|z|<1$ on the slit domain with $h(0)=0$, for a suitable length of slit we shall have $\left|h^{\prime}(0)\right|=\lambda$. Further the least upper bound of $|h(z)|$ for $|z|<1$ will be the same as the least upper bound of $|g(z)|$ for $|z|<1$ and this is at least as large as the same quantity for $f(z)$.

Now, if for $f(z)$ a univalent function in $C$ we have

$$
\underset{|z|<1}{\text { l.u.b. }}|f(z)|=M \text {, }
$$

for the modulus $\mu$ of the boundary point of the image of $|z|<1$ under $f$ closest to the origin we have $\mu \leqq 1 / M$ so $M \leqq 1 / \mu$. Thus $P(\lambda) \leqq(\mu(\lambda))^{-1}$. We shall now show that the precise value is $P(\lambda)=(\mu(\lambda))^{-1}$.

First we observe by an argument similar to that of the first paragraph of this section that for $\lambda^{\prime} \geqq \lambda, P\left(\lambda^{\prime}\right) \leqq P(\lambda)$.

Let $t$ be the value corresponding to $\lambda$ as in $\S 4$. We may assume $t>0, \lambda<1$ since in the special case excluded the result is evident. Let us denote by $D(t, \epsilon)$ the domain obtained from $D(t)$ by shortening the slit from $-i l$ to $-i m$ to a slit from $-i l$ to $-i(m+\epsilon), 0<\epsilon<l-m$. Let $f(z ; t, \epsilon)$ be the function mapping $|z|<1$ on to $D(t, \epsilon)$ such that $f(0 ; t, \epsilon)=0, f^{\prime}(0 ; t, \epsilon)>0$. Clearly $f^{\prime}(0 ; t, \epsilon)>\lambda$. Let $U(\sigma)$ denote the open set of points whose distance from the segment joining $i / l$ to $i /(m+\epsilon)$ is less than $\sigma$. Let $U^{*}(\sigma)$ be the image of $U(\sigma)$ by the transformation $w^{*}=1 / w$. Let

$$
D(t, \epsilon, \sigma)=(D(t, \epsilon) \cup U(\sigma))-\bar{U}^{*}(\sigma) .
$$

For $\sigma$ sufficiently small this is a simply-connected domain containing the point $w=0$. Let $f(z ; t, \epsilon, \sigma)$ be the function with $f(0 ; t, \epsilon, \sigma)=0, f^{\prime}(0 ; t, \epsilon, \sigma)>0$ 
mapping $|z|<1$ onto $D(t, \epsilon, \sigma)$. By the general theory of kernels of domains [1], $f(z ; t, \epsilon, \sigma)$ converges to $f(z ; t, \epsilon)$ as $\sigma$ tends to zero. Thus $f^{\prime}(0 ; t, \epsilon, \sigma)>\lambda$ for $\sigma$ sufficiently small. However

$$
\underset{|z|<1}{\operatorname{l.u} . b .}|f(z ; t, \epsilon, \sigma)|=(m+\epsilon)^{-1}+\sigma .
$$

Thus by the monotone property given above

$$
P(\lambda) \geqq(m+\epsilon)^{-1}+\sigma
$$

for $\epsilon, \sigma$ positive, sufficiently small. Hence

$$
P(\lambda) \geqq m^{-1}=(\mu(\lambda))^{-1} .
$$

We summarize these statements in our principal result.

THEOREM 3. If $P(\lambda)$ is the best possible uniform bound for functions in $C(\lambda)$, $P(\lambda)=(\mu(\lambda))^{-1}$. There is no function $f(z)$ in $C(\lambda)$ having $P(\lambda)$ as least upper bound of $|f(z)|,|z|<1$, except for $\lambda=1$.

The final statement of Theorem 3 follows from the uniqueness part of Theorem 2, since for such a function $f(z)$ the modulus $\mu$ of the boundary point of the image of $|z|<1$ under $f$ closest to the origin would satisfy $\mu \leqq \mu(\lambda)$, thus $\mu=\mu(\lambda)$ and $f(z)$ would be $e^{i \theta} F\left(e^{i \psi} z ; \lambda\right), \theta, \psi$ real. However $\left|F\left(e^{i \psi} z ; \lambda\right)\right|$, for $|z|<1$, has least upper bound strictly less than $(\mu(\lambda))^{-1}$ except when $\lambda=1$.

\section{BIBLIOGRAPHY}

1. C. Carathéodory, Untersuchungen ïber die konformen Abbildungen von festen und veränderlichen Gebieten, Math. Ann. vol. 72 (1912) pp. 107-144.

2. James A. Jenkins, Some problems in conformal mapping, Trans. Amer. Math. Soc. vol. 67 (1949) pp. 327-350.

3. - Remarks on "Some problems in conformal mapping," Proc. Amer. Math. Soc. vol. 3 (1952) pp. 147-151.

4. - Symmetrization results for some conformal invariants, Amer. J. Math. vol. 75 (1953) pp. 510-522.

5. - On Bieberbach-Eilenberg functions, Trans. Amer. Math. Soc. vol. 76 (1954) pp. 389-396.

6. - On the local structure of the trajectories of a quadratic differential, Proc. Amer. Math. Soc. vol. 5 (1954) pp. 357-362.

7. - Some unigueness results in the theory of symmetrization, Ann. of Math. vol. 61 (1955) pp. 106-115.

8. G. Polya and G. Szegö, Isoperimetric inequalities in mathematical physics, Annals of Mathematics Studies, No. 27, Princeton University Press, 1951.

9. W. Rogosinski, On a theorem of Bieberbach-Eilenberg, J. London Math. Soc. vol. 14 (1939) pp. 4-11.

10. A. C. Schaeffer and D. C. Spencer, Coefficient regions for schlicht functions, Amer. Math. Soc. Colloquium Publications, vol. 35, 1950.

Johns Hopkins University,

BAltimore, MD. 